\title{
Synthesis, characterization and low frequency a.c. conduction of polyaniline/fly ash composites
}

\author{
S C RAGHAVENDRA, SYED KHASIM ${ }^{\dagger}$, M REVANASIDDAPPA \\ M V N AMBIKA PRASAD ${ }^{\dagger}$ and A B KULKARNI* \\ Department of Applied Electronics, ${ }^{\dagger}$ Department of Physics, "Department of Chemistry, Gulbarga University, \\ Gulbarga 585 106, India
}

MS received 2 June 2003; revised 16 September 2003

\begin{abstract}
In situ polymerization of aniline was carried out in the presence of fly ash (FA) to synthesize polyaniline/fly ash (PANI/FA) composites. The PANI/FA composites have been synthesized with various compositions $(15,20,30$ and $40 \mathrm{wt} \%)$ of $\mathrm{FA}$ in PANI. The composites, thus synthesized have been characterized by infrared spectroscopy and X-ray diffraction. The morphology of these samples was studied by scanning electron microscopy. Further the a.c. conductivity of these composites have been investigated in the frequency range $10^{2}-10^{6} \mathrm{~Hz}$. The presence of polarons and bipolarons are made responsible for frequency dependence of a.c. conductivity in these composites. The Cole-Cole plots indicate clear shift in the distribution of relaxation times as the wt\% of FA in PANI changes. These composites show almost symmetric semicircles of Cole-Cole plots indicating the Debye-type relaxation in their polarization response.
\end{abstract}

Keywords. Conductivity; polarons; polyaniline; fly ash.

\section{Introduction}

The conducting polymers have emerged as a new class of materials because of their unique electrical, optical and chemical properties. By proper doping the conductivity of these materials can be varied from semiconducting to metallic regime, which has offered new concept of charge transport mechanism. Conductive polyaniline (PANI) has been studied extensively because of its ease of synthesis in aqueous media, its environmental stability and special electrical and other properties. PANI has become a suitable candidate for variety of technological applications (Matsunaga et al 1990; Gustafsson et al 1992; Olcani et al 1993), such as solar cells, electromagnetic shielding, electrodes for rechargeable batteries, sensors, etc. Preparation of composites of conducting polymer (PANI) has been considered to provide a suitable solution to the processibility problem. These composites have the ability to enhance their material properties with desirable mechanical and physical characteristics. One way of making these composites involves synthesizing the conductive polymer inside the matrices of conventional polymers using chemical (Mohammadi et al 1993; Chakraborty et al 1999) or electrochemical (Niwa and Tamamura 1984; Yasoniiya et al 1986; Wang et al 1990) polymerization. There are many reports pertaining to the chemical preparation of conductive polymer composites without using any insulating polymers (Gill et al 1992; Maeda and

*Author for correspondence
Armes 1995). The most preferred method for synthesis of PANI composites is to use either $\mathrm{HCl}$ or $\mathrm{H}_{2} \mathrm{SO}_{4}$ with ammonium peroxydisulfate as an oxidant (MacDiarmid et al 1985; Armes and Miller 1988). In these approaches, monomer is polymerized in the presence of commercially available inorganic acid particles which act as colloidal substrate for the precipitating polymer nuclei leading to the formation of conducting polymer-inorganic oxide composites.

Fly ash (FA) is a coal product generated from coal fired thermal power stations. It is a alkaline grey powder with $\mathrm{pH}$ ranging from 9-9.9. Large number of coal fired power plants all over the world, disposes a huge quantity of FA causing serious environmental problems (Sahu 1994). Less than half of the ash is used as a raw material for concrete manufacturing and construction; the remaining is directly dumped on land side as land fill or simply piled up. Due to environmental regulations, new ways of utilizing FA have to be explored in order to safeguard the environment and provide useful ways for its disposal. Hence, there is considerable interest in utilization of FA as raw material. For the first time, FA was used in the preparation of cordierite (Sampathkumar et al 1995). Because of the presence of $\mathrm{SiO}_{2}$ and $\mathrm{Al}_{2} \mathrm{O}_{3}$ in high proportions the FA was used to synthesize zeolites (LaRosa et al 1992; Shigemoto et al 1993). FA was treated hydrothermally and the performance of this material as cracking catalyst was investigated with heavy oil fraction as the cracking feed stock (Ozha and Pradhan 2001). On the other hand, there were many experimental analyses on FA to undertake basic compositional, physical and chemical proper- 
ties for technical studies and applications (Cumpston et al 1992; Sajwan et al 1995).

Conducting polymer composites with some suitable composition of one or more insulating materials lead to desirable properties. These materials are especially important owing to their bridging role between the world of conducting polymers and that of nanoparticles. For application of conducting polymers, knowing how these conducting polymer composites will affect the behaviour in an electric field is a long-standing problem and of great importance. But very little is known about the dielectric properties of conducting polymer associated with the conducting mechanism. Dielectric spectroscopy has been found to be a valuable experimental tool for understanding the phenomena of charge transport in conducting polymers. Low frequency conductivity and dielectric relaxation measurements especially have proven to be valuable in giving additional information on the conduction mechanism that d.c. conductivity measurement alone does not provide. In this work a new potential use of FA has been proposed. The high dielectric constant FA (Raghavendra et al 2002a) has been used for the synthesis of polyaniline/fly ash (PANI/FA) composites. Also qualitative analysis of chemical composition of FA has been reported. Here, we report our observation of the morphology and conducting properties of PANI/FA composites, synthesized by chemical oxidation process.

\section{Experimental}

\subsection{Synthesis of PANI/FA composites}

Aniline (AR grade) was purified by distillation before use and ammonium peroxydisulfate $\left[\left(\mathrm{NH}_{4}\right)_{2} \mathrm{~S}_{2} \mathrm{O}_{8}\right], \mathrm{HCl}$ were used as received. A fine fresh, clean and pure FA powder was collected from the Raichur Thermal Power Station, Raichur, Karnataka. The cenosphere type FA was grounded and sintered at $600^{\circ} \mathrm{C}$ to remove moisture content and impurities present. Chemically, the FA was silica to an extent of $55-70 \%$ followed by alumina, 10-18\%, iron oxide, 6-20\% and lime magnesia and alkalis varied between 1 and 5\% each. FA also contains elements like $\mathrm{Cu}$, $\mathrm{Pb}, \mathrm{Cd}, \mathrm{Ag}, \mathrm{Mn}, \mathrm{Ti}, \mathrm{Na}, \mathrm{Mo}, \mathrm{S}, \mathrm{P}, \mathrm{Zn}$ and $\mathrm{Cl}$ in different concentrations (Vogel 1969; Raghavendra et al 2002a).

$0.1 \mathrm{~mol}$ of aniline was dissolved in $1000 \mathrm{ml}$ of $2 \mathrm{M}$ $\mathrm{HCl}$ to form polyaniline (PANI). The varied wt\% of fly ash powder $(15,20,30$ and $40 \%)$ was added to the PANI solution with vigorous stirring in order to keep the FA powder suspended in the solution. $0 \cdot 1 \mathrm{~mol}$ of $\left[\left(\mathrm{NH}_{4}\right)_{2} \mathrm{~S}_{2} \mathrm{O}_{8}\right]$ was added slowly with continuous stirring, which acts as the oxidant. The reaction mixture was agitated continuously for another $8 \mathrm{~h}$. In this process, the solution was always kept at $5^{\circ} \mathrm{C}$. The precipitate formed was collected by filtration and washed with distilled water and acetone until the filtrate became colourless. In the mean time, the precipitate was also tested to ensure that there were no free $\mathrm{SO}_{4}^{2-}$ ions in the filtrate by using $\mathrm{BaCl}_{2}$ solution. Any unreacted aniline in the composites formed was removed by acetone. After washing, the precipitate was dried at room temperature.

PANI/FA composites were prepared in $4 \mathrm{wt} \%$ ratio, in which the concentration of FA was varied as $15 \%, 20 \%$, $30 \%$ and $40 \%$ by weight, respectively. These samples were pressed in the form of circular pellets of diameter, $0.8 \mathrm{~cm}$ and thickness, $0 \cdot 3 \mathrm{~cm}$. The electrical measurements on these samples were made using the silver paint as electrodes on both sides.

\subsection{Measurements}

The electrical conductivity of the PANI/FA composites were measured using Hewlett-Packard impedance analyser $4192-\mathrm{A}$ in the frequency range $10^{2}-10^{6} \mathrm{~Hz}$ at room temperature. The infrared (IR) spectra of the composites were recorded on Perkin Elmer (model 783) IR spectrometer in $\mathrm{KBr}$ medium at room temperature, in the region 4000$450 \mathrm{~cm}^{-1}$. X-ray diffraction patterns of the PANI/FA composites were taken with Philips X-ray diffractometer using CuK $\alpha$ radiation $(\lambda=1.5406 \AA)$. The powder morphology of PANI and PANI/FA samples in the form of pellets (to measure grain size) was investigated using Philips XL 30 ESEM scanning electron microscope (SEM).

\section{Results and discussion}

\subsection{Infrared spectra}

Figure 1a shows the IR spectrum of PANI, where the \% of transmittance is plotted as a function of wave number $\left(\mathrm{cm}^{-1}\right)$. The characteristic IR peak at 1559 and $1484 \mathrm{~cm}^{-1}$ are due to the presence of quinoid and benzenoid rings, respectively and are clear indication of these two states in the polymer chain (Radhakrishnan and Deshpande 2002). Figure 1b shows the IR spectrum of FA. The characteristic IR peak observed at $1094 \mathrm{~cm}^{-1}$ may be attributed to the presence of silica (Chowdhury et al 2002). This peak confirms the highest $\%$ of silica in the FA, which is confirmed by chemical analysis (Raghavendra et al 2002a). The broadening of the peak may be due to the merging of IR peaks corresponding to the other metal oxides present in the FA. The IR spectra of PANI composite (figure 1c) in presence of FA exhibit new absorption peaks distinctly at $1591,1497,1299,1134$ and $798 \mathrm{~cm}^{-1}$ which are assignable to the presence of various metal oxides in the composite (Raghavendra et al 2002a). The IR spectra of other composites (PANI with 10, 15, 20 and $30 \mathrm{wt} \%$ of FA) do not show much variation in the characteristic peaks.

\section{$3.2 X$-ray diffraction}

Figure 2a shows X-ray diffraction pattern of PANI ( 0 wt $\%$ of FA). Diffraction pattern of PANI have a broad 
peak at about $2 \theta=25.92^{\circ}$, which is a characteristic peak of PANI (Wan et al 1994; Wan and Li 1998). Studies on XRD patterns of PANI are scarce in the literature (Rajendra Prasad and Munichandriah 2002). Figure 2b shows XRD pattern of FA. The FA consists of quartz and mullite as crystalline phases as indicated by X-ray patterns and some quantity of glassy phase (Sampathkumar et al 1995). Figure 2c shows the XRD pattern for PANI/ FA composite with $40 \mathrm{wt} \%$ of FA in PANI. The diffraction pattern of PANI/FA composite shows a sharp peak at about $2 \theta=26 \cdot 65^{\circ}$. The characteristic peak of PANI has been shifted slightly in these composites. This peak in PANI/FA composite shows semi-crystalline nature.

\subsection{Scanning electron micrograph}

SEM of conducting PANI ( 0 wt $\%$ of FA) synthesized by chemical oxidative method is shown in figure $3 \mathrm{a}$. It is clear from the SEM of PANI that the sample is smooth and homogeneous, which is confirmed by XRD patterns. Conducting polymers are very sensitive to the tempera-

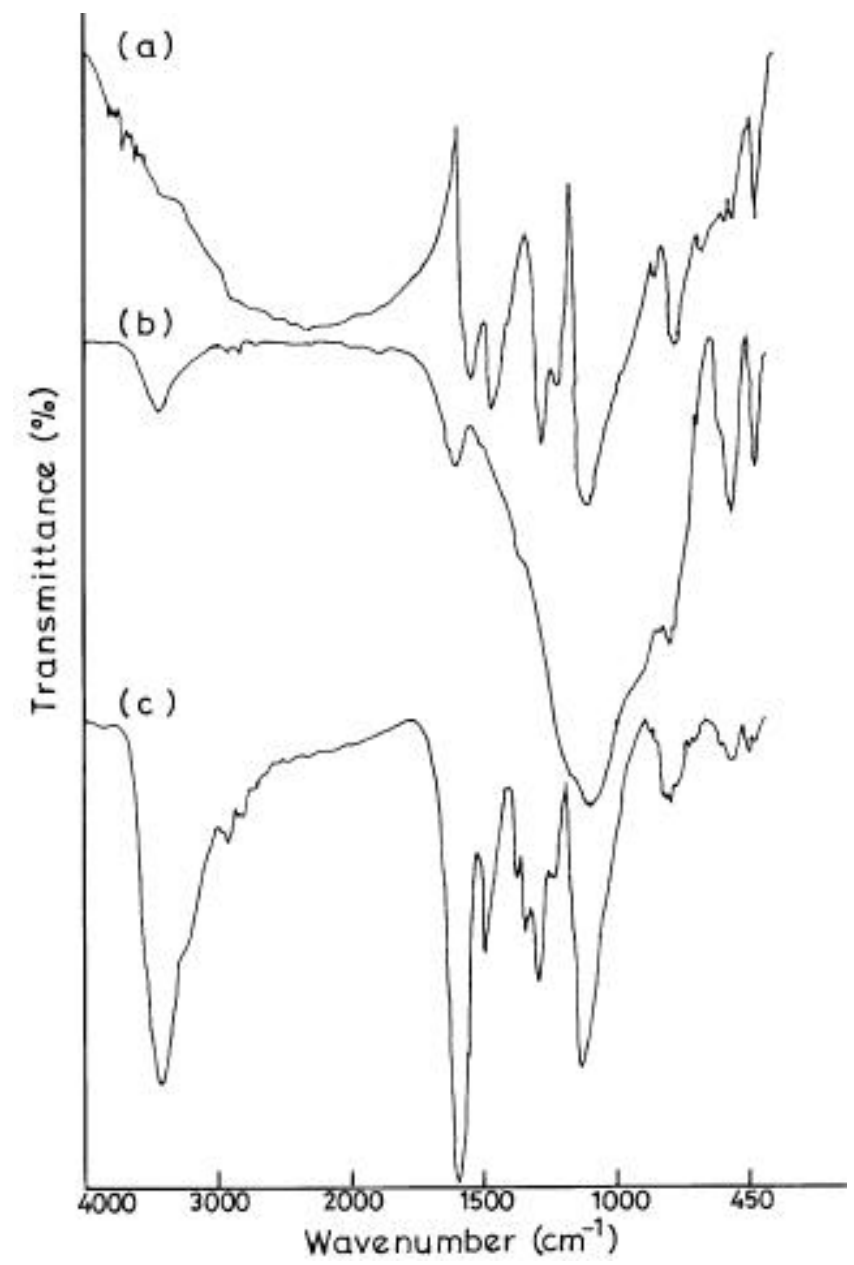

Figure 1. Infrared spectra of (a) polyaniline, (b) fly ash and (c) PANI/FA composite (with $40 \mathrm{wt} \%$ of FA in PANI). ture. Due to the interaction between electron and sample, considerable amount of heat is generated which causes the development of small cracks during SEM recording. The contrast in the image is a result of differences in the scattering from different surface areas due to geometrical differences (Kumar and Chandra 2001).

SEM of PANI/FA composite with $40 \mathrm{wt} \%$ of FA in PANI is shown in figure $3 \mathrm{~b}$. A very high magnification of SEM image shows the presence of spherical FA particles (cenospheres) in PANI, which are homogeneously distributed throughout the composites, which is also confirmed from XRD studies. It is for the first time such a beautiful distribution of cenospheres is observed which looks as if the beads are floating over the water surface. These cenospheres show a large variation in their dimensions. The lowest diameter of cenospheres recorded was $270 \mathrm{~nm}$ and highest with a diameter of $890 \mathrm{~nm}$. The particle size of raw FA varies between 0.4 and $6 \mu \mathrm{m}$, the average particle size is about $3.2 \mu \mathrm{m}$. The variation in the dimensions of cenospheres depends on the conditions of the thermal plants during which the FA has been expelled out. Such a large variation in the dimensions of

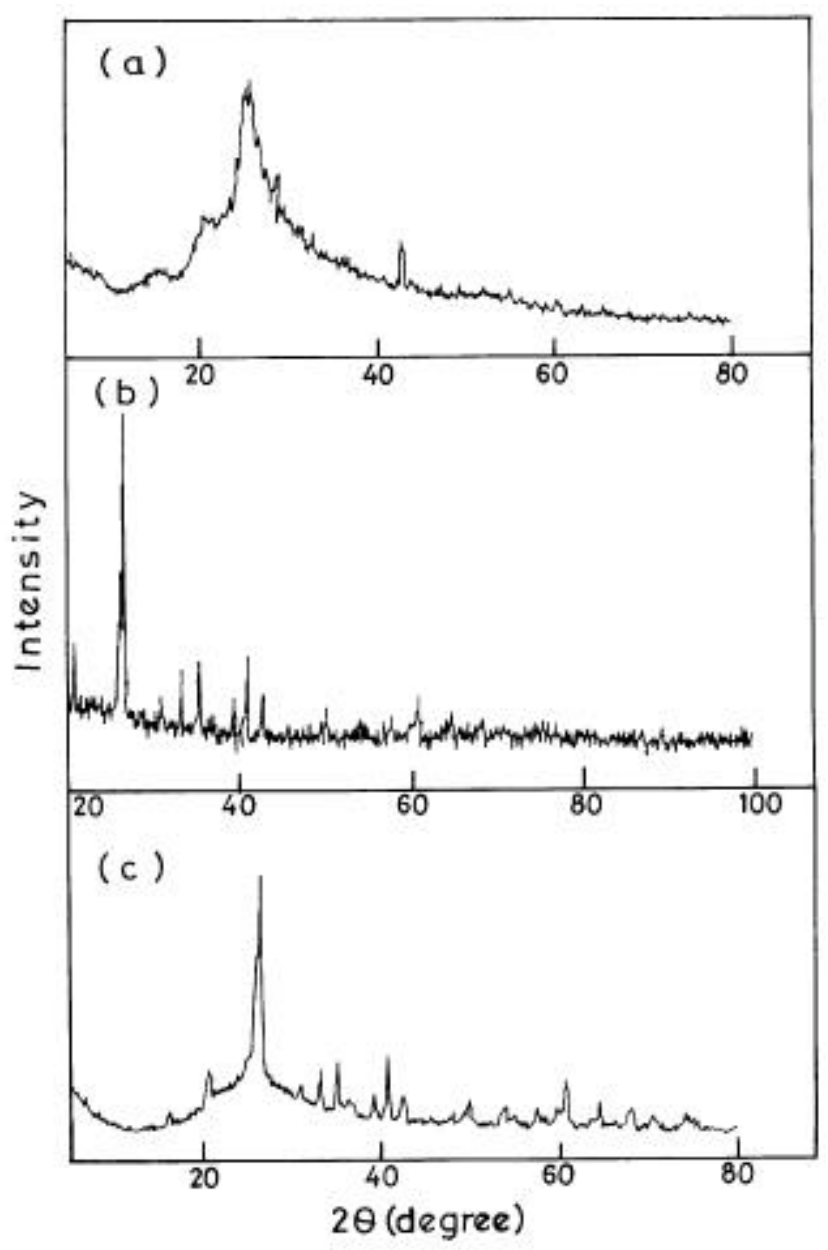

Figure 2. XRD spectra of (a) polyaniline, (b) raw fly ash and (c) PANI/FA composite (with $40 \mathrm{wt} \%$ of FA in PANI). 

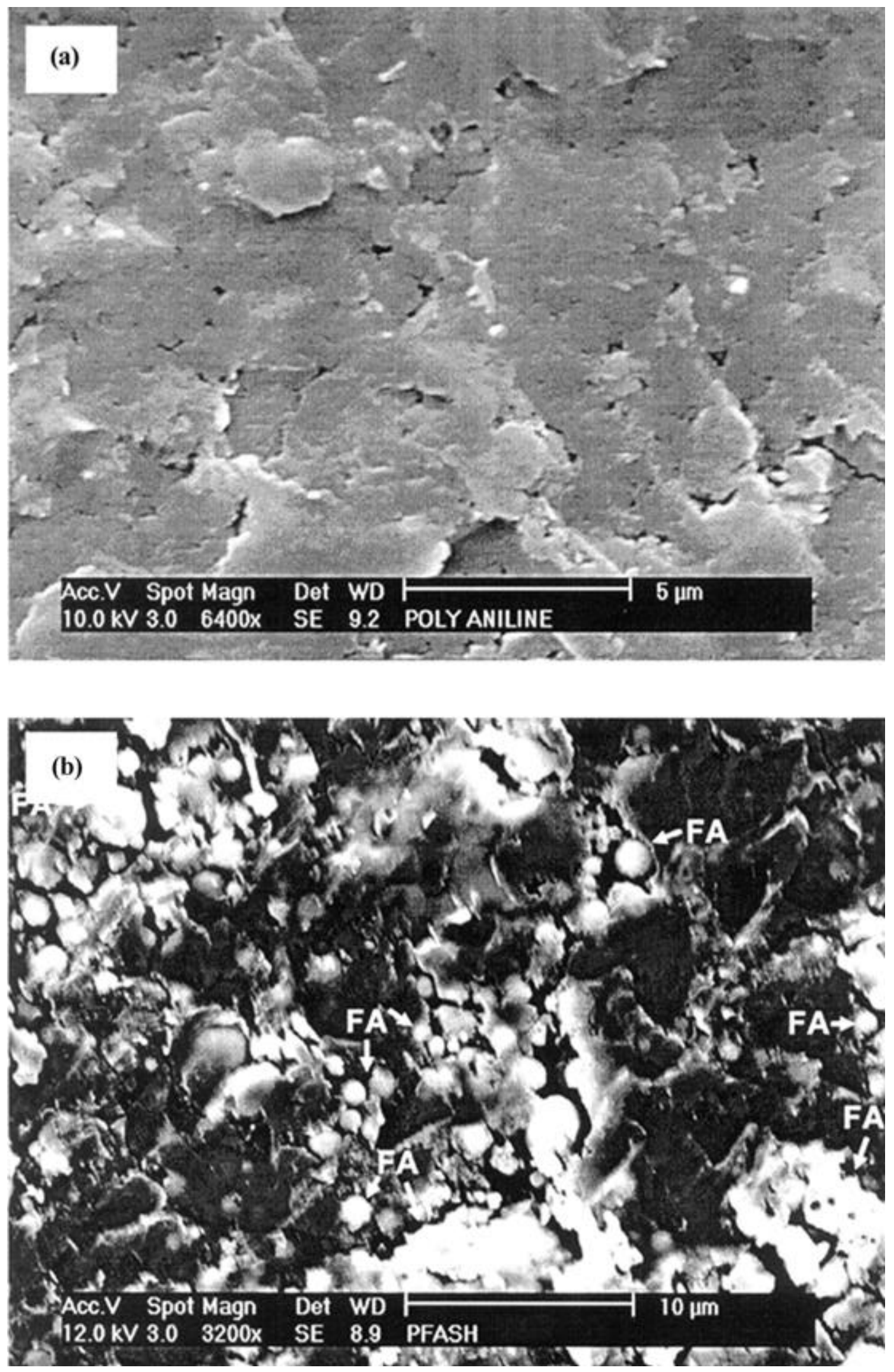

Figure 3. Scanning electron micrograph of (a) PANI $(\times 6400)$ and $($ b) PANI/FA composite (with $40 \mathrm{wt} \%$ of FA in PANI) $(\times 3200)$ (arrow marks show the location of FA). 
cenospheres has a strong influence on the various electrical properties of the composites. Since the particles of FA are spherical in shape, the observed porosity in these composites is less than the other PANI composites (Chowdhury et al 2002).

\subsection{Electrical conductivity}

The effect of FA content on the electrical conductivity of PANI/FA composites is plotted in figure 4. In these plots, the low frequency behaviour (up to $10^{4} \mathrm{~Hz}$ ) of all the composites looks like a straight line typical of hopping conduction (Berner et al 1999). The absolute conductivity for individual samples increases as a function of frequency except for the composite with $20 \mathrm{wt} \%$ of FA. The a.c. electrical conductivity of PANI ( $0 \mathrm{wt} \%$ of FA) (figure 4 , inset) has the order of $10^{-4} \mathrm{~S} / \mathrm{cm}$. For the PANI/FA composites the conductivity values abruptly change the order of magnitude $\left(10^{-6} \mathrm{~S} / \mathrm{cm}\right)$. As the wt $\%$ of FA in PANI increases the absolute conductivity shifts to lower scales except for the $20 \mathrm{wt} \%$ PANI/FA composite. This shift is due to the electrical charges being displaced inside the polymer (stronger localization) and/or due to their lower concentration. The decrease in conductivity, by increase in wt\% of FA may be due to particle blockage conduction path by FA (nano size particle) embedded in PANI matrix (Su and Kuramoto 2000). Also, increase in wt\% of FA leads to an increasing inter chain distance, which makes hopping between chains more difficult, re-

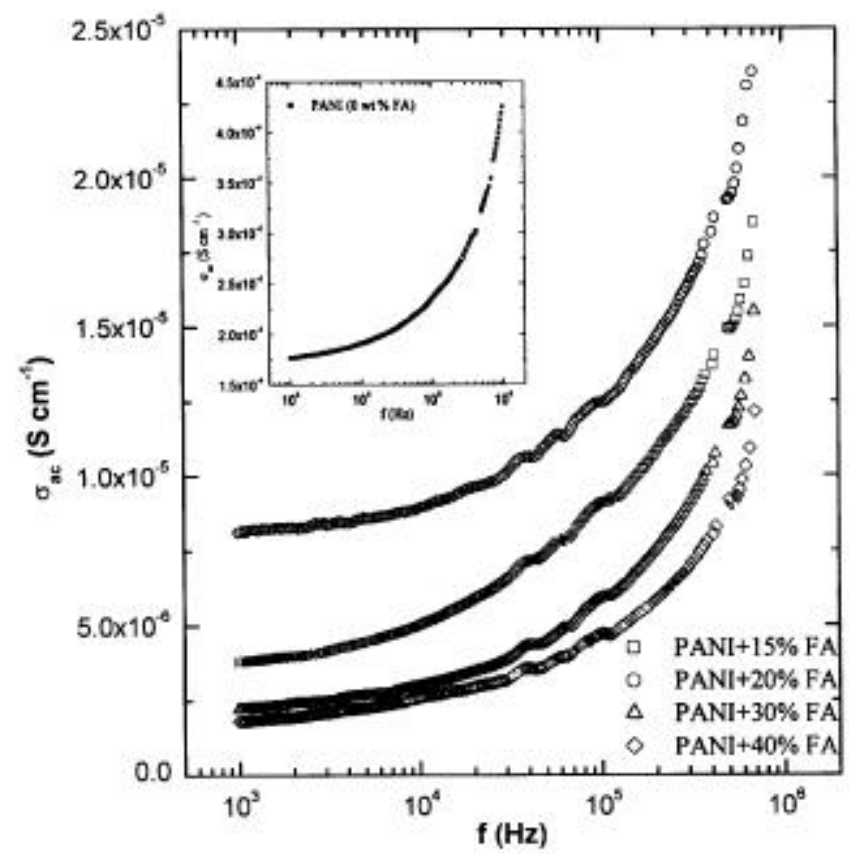

Figure 4. Frequency dependence of conductivity $\left(\sigma_{\text {a.c. }}\right)$ for PANI/FA composites for various weight $\%$ of FA (Inset: Frequency dependence of $\left(\sigma_{\text {a.c. }}\right)$ for PANI). sulting in reduction of conductivity. However, at higher frequencies the conductivities of all the samples (with different wt $\%$ of FA) merge to show similar frequency dependence, indicating the formation of excess charge carriers (polarons and bipolarons). Figure 5 shows the conductivity of PANI/FA composites at different wt $\%$ of FA at frequency, $f=10 \mathrm{kHz}$. It is clear from the figure that there is a $10^{-2}$ order difference in the conductivity values for PANI and PANI/FA composites. The conductivity of PANI/FA composites decreases with increase of FA content. From PANI/FA with $20 \mathrm{wt} \%$ of FA content onwards the conductivity decreases with excess of FA content. Conductivity of all the samples is of the same order $\left(10^{-6} \mathrm{~S} / \mathrm{cm}\right)$. The special observation made here is the large variation in conductivity values of PANI and PANI/FA composites. Su and Kuramoto (2000) reported the nano composite films of PANI-DBSA/TiO ${ }_{2}-\mathrm{DBSA}$ at different contents of $\mathrm{TiO}_{2}$. They observed that the conductivity of the nano composite films increases slightly with increasing $\mathrm{TiO}_{2}$ content and decreases with excess of $\mathrm{TiO}_{2}$ content. The decrease in the conductivity observed in the PANI/FA composites is typically due to the presence of various other metallic oxides, metals and also due to the complicated structure of FA particles. From IR and XRD studies, the presence of various oxides and different crystalline phases (quartz, mullite and glass) in FA are confirmed. Due to the insufficient thermal energy to excite the charge carriers in FA (Raghavendra et al 2002b) the conductivity values of PANI/FA decreases sharply to $10^{-6} \mathrm{~S} / \mathrm{cm}$ than pure PANI. If these composites can be

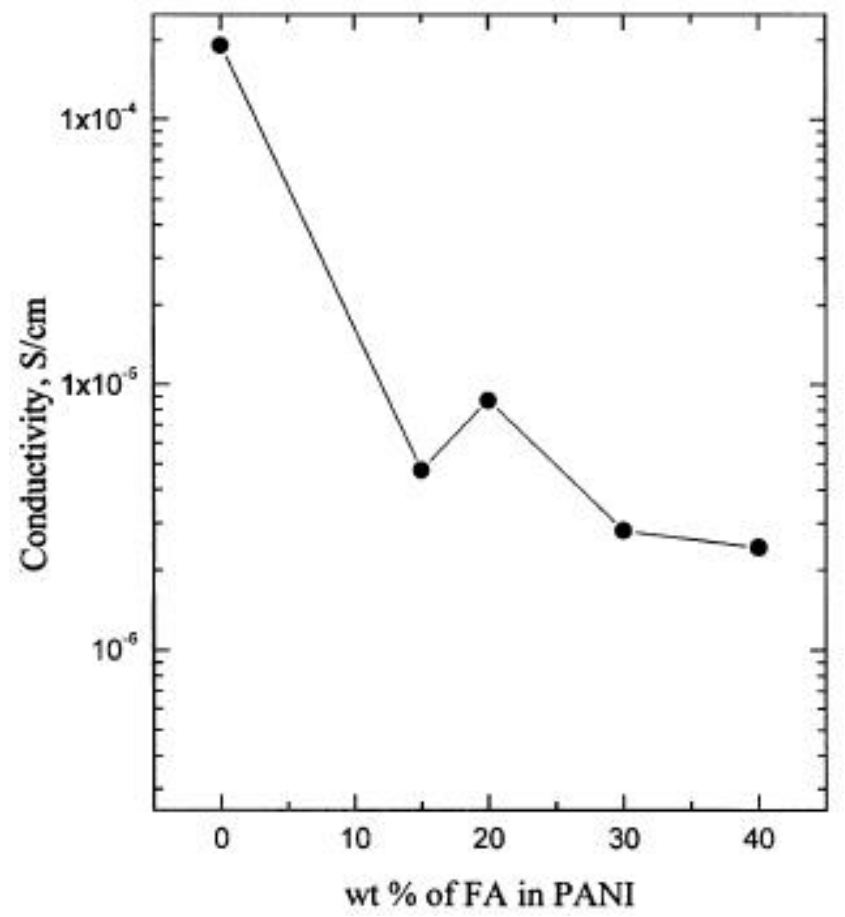

Figure 5. The conductivity of PANI/FA composites for different wt $\%$ of FA in PANI at frequency, $10 \mathrm{kHz}$. 
studied at higher temperatures better effects could be observed which may be useful in applications. The temperature sensitivity of PANI and its composites puts a limitation for such studies.

Figure 6 shows Cole-Cole plots of respective PANIFA composites. A beautiful shift in the peaks is observed for increasing weight $\%$ of FA in PANI. The relaxation time $(\tau)$ of the individual PANI/FA composites is calculated using the Cole-Cole plots by the relation

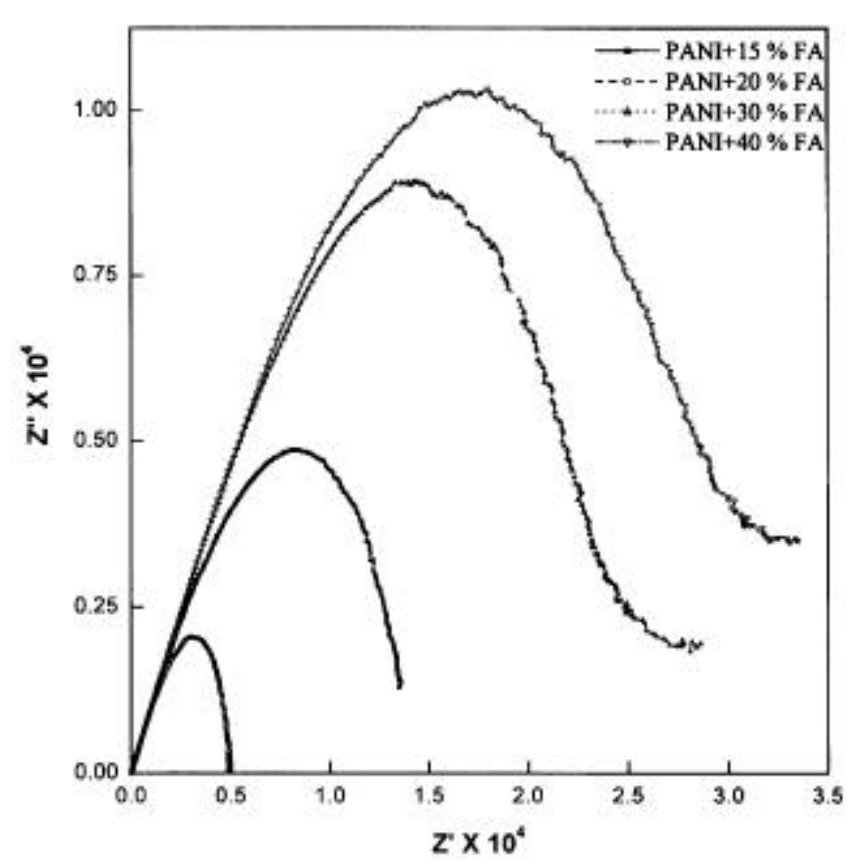

Figure 6. The Cole-Cole plots for PANI/FA composites for various weight $\%$ of FA in PANI.

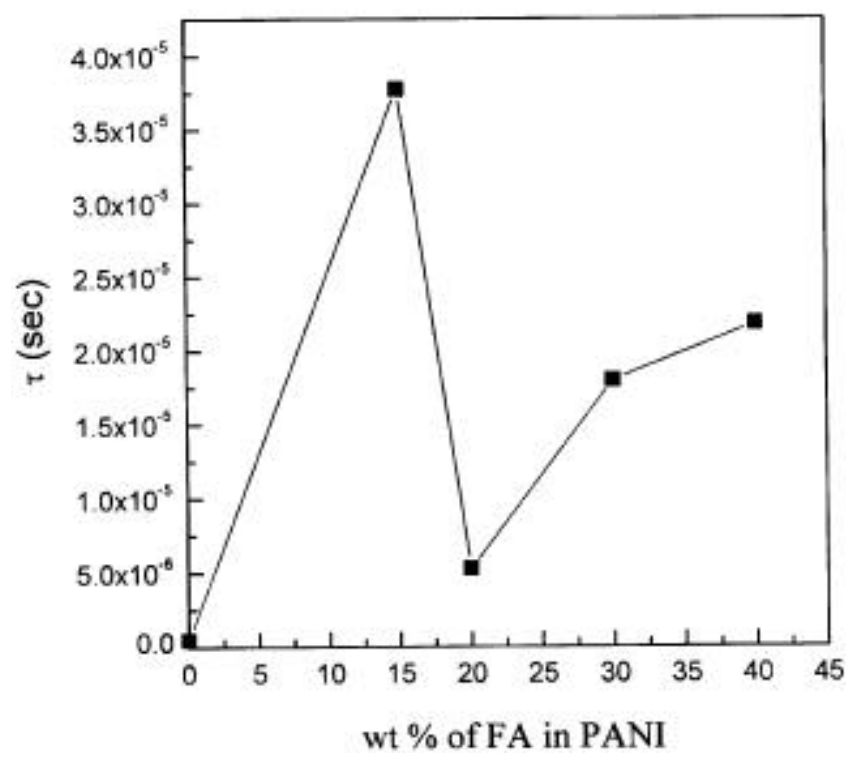

Figure 7. The distribution of relaxation times for various PANI/FA composites.

$$
\tau=1 / 2 \pi f_{\mathrm{p}},
$$

where $f_{\mathrm{p}}$ is the value of frequency for maximum peak position of Cole-Cole plot. The analysis of Cole-Cole plots indicates a clear increase in the distribution of relaxation times as the weight \% of FA in PANI increases. The distribution of relaxation times for various PANI-FA composites are as shown in figure 7 . It is observed from these plots that the composites show almost symmetric semicircles indicating the Debye-type relaxation.

\section{Conclusions}

We have synthesized conducting polyaniline/fly ash (PANI/ FA) composites by in situ polymerization in the presence of FA. The PANI/FA composites with varying wt $\%$ of FA have been characterized by IR, XRD and SEM techniques. The silica is present in excess followed by various other metal oxides in the composites and is confirmed by IR studies. The PANI/FA composites show semi-crystalline nature, whereas the PANI synthesized is amorphous in nature. The SEM photograph of PANI/FA composite with $40 \mathrm{wt} \%$ of FA clearly show the presence of cenospheres (measuring in $\mathrm{nm}$ scale). Our results on a.c. conductivity for various weight $\%$ of FA in PANI are interpreted in terms of the formation of polarons and bipolarons and also due to increase in the chain length between which the charge carriers hop. The PANI/FA composites show a sudden fall in their conductivity $\left(10^{-6} \mathrm{~S} / \mathrm{cm}\right)$ in comparison with pure PANI $\left(10^{-4} \mathrm{~S} / \mathrm{cm}\right)$. PANI/FA with $20 \mathrm{wt} \%$ of FA shows a higher value of conductivity among other composites with varying content of FA. Cole-Cole plots and distribution of relaxation times for these composites show a Debye-type relaxation. Through this study we have made a successful effort towards better utility of fly ash, which is considered to be an environmental waste. These composites may become promising candidates for advanced materials to be used in the high-technology industries in the future. Also these materials can reduce the cost due to usage of FA. Work on the exploration of these composites at higher frequencies $(\mathrm{GHz})$ is currently underway.

\section{Acknowledgements}

The authors wish to thank Mr Basavaraj Angadi, MRC, IISc, Bangalore, for valuable discussions. One of the authors (SCR) thanks the Council of Scientific and Industrial Research (CSIR), New Delhi, for the award of a Senior Research Fellowship.

\section{References}

Armes S P and Miller J F 1988 Synth. Met. 22385

Berner D, Travers J P and Rannou P 1999 Synth. Met. 101836 
Chakraborty M, Mukherjee D C and Mandal B M 1999 Synth. Met. 98193

Chowdhury A N, Yousuf M A, Rahman M M and Hassan A Q M Q 2002 Indian J. Chem. A41 1562

Cumpston B, Shadman F and Risbud S 1992 J. Mater. Sci. 27 1781

Gill M, Mykytiuk J, Armes S P, Edward J L, Yeates T, Moreland P J and Mollet C 1992 J. Chem. Soc.: Chem. Commun. 108

Gustafsson G, Cao Y, Treacy G M, Klavetter F, Colaneri N and Heeger A J 1992 Nature 357477

Kumar D and Chandra R 2001 Ind. J. Engg. Mater. Sci. 8209

LaRosa J L, Kwan S and Grutzeek M W 1992 J. Am. Ceram. Soc. 751574

MacDiarmid A G, Chiang J C, Halpern M, Huang W S, Mu S L, Somasiri N L, Wu W and Yaniger S I 1985 Mol. Cryst. Liq. Cryst. 121173

Maeda S and Armes S P 1995 Chem. Mater. 7171

Matsunaga T, Daifuku H, Nakajima T and Gawa-goe T 1990 Polym. Adv. Tech. 13

Mohammadi A, Lundstrom I, Inganas $\mathrm{O}$ and Salaneck W R 1993 Polymer 31395

Niwa O and Tamamura T 1984 J. Chem. Soc. Chem. Commun. 817

Ojha K and Pradhan N C 2001 Indian J. Engg. Mater. Sci. 8 100
Olcani A, Abe M, Ezoe M, Doi T, Miyata T and Miyake A 1993 Synth. Met. 573969

Radhakrishnan S and Deshpande S D 2002 Sensors 2185

Raghavendra S C, Raibagkar R L and Kulkarni A B 2002a Bull. Mater. Sci. 2537

Raghavendra S C, Raibagkar R L and Kulkarni A B 2002b Indian J. Pure Appl. Phys. 40367

Rajendra Prasad K and Munichandraiah N 2002 Synth. Met. 13017

Sahu K C 1994 Power plant pollution: Cost of coal combustion, Survey of the Environment, The Hindu

Sajwan K S, Haroldornes W and Youngbold T 1995 J. Environ. Sci. Hlth. 301327

Sampathkumar N N, Umarji A M and Chandrashekar B K 1995 Mater. Res. Bull. 301107

Shigemoto K, Hayashi H and Miyaura K 1993 J. Mater. Sci. 28 4581

Su S J and Kuramoto N 2000 Synth. Met. 114147

Vogel A I 1969 A text book of quantitative inorganic analysis (London: Longman)

Wan M and Li J 1998 J. Polym. Sci. A36 2799

Wan M, Li M, Li J and Liu Z 1994 J. Appl. Polym. Sci. 53131

Wang H L, Toppare L and Fernandez J E 1990 Macromolecules 231053

Yosoniiya R, Hirata M, Haga Y, An H and Saki M 1986 Makromol. Chem. Rapid Commun. 7697 